\begin{tabular}{|c|l|}
\hline Title & $\begin{array}{l}\text { Structure and dynamics of a magnetic neutral loop discharge plasma described using electron motion in a quadrupole } \\
\text { magnetic field }\end{array}$ \\
\hline Author(s) & Osaga, Tsuyoshi; Sugawara, Hirotake; Sakurai, Y ohei \\
\hline Citation & $\begin{array}{l}\text { Plasma Sources Science and Technology, 20/6), 065003 } \\
\text { https:/doi.org/L0.1088/0963_0252/20/6065003 }\end{array}$ \\
\hline Issue Date & 2011-12 \\
\hline Doc URL & http://hdl.handle.net/2115/48133 \\
\hline Rights & ○ 2011 IOP Publishing Ltd \\
\hline Type & article (author version) \\
\hline File Information & PSST 20-6_065003.pdf \\
\hline
\end{tabular}

Instructions for use 


\title{
Structure and dynamics of a magnetic neutral loop discharge plasma described using electron motion in a quadrupole magnetic field
}

\author{
Tsuyoshi Osaga, Hirotake Sugawara and Yohei Sakurai \\ Division of Electronics for Informatics, Graduate School of Information Science and \\ Technology, Hokkaido University, Sapporo 060-0814 Japan \\ E-mail: sugawara@ist.hokudai.ac.jp
}

\begin{abstract}
Electron motion in a $\mathrm{CF}_{4}$ magnetic neutral loop discharge (NLD) plasma was simulated using a Monte Carlo method. The spatial distribution of electrons illustrated the fundamental structure of the NLD plasma, and its dynamics were depicted from the distributions of the mean electron energy, the electron energy gain and the azimuthal electron flux. The peak of mean electron energy appeared at the neutral loop (NL), which confirmed that electrons gain energy near this loop. High mean electron energy was observed not only near the NL but also along the separatrices of the quadrupole magnetic field. Energetic electrons were transported along the separatrices and induced ionization at those locations. However, the electron distribution had valleys along the separatrices, because electrons accelerated near the NL were likely to leave this region where the binding of the magnetic field is weak. The azimuthal electron flux representing the loop plasma current showed that the electron conduction path around the NL, which has conventionally been modelled as a ring conductor, has a particular directionality due to the rectification effect of antiparallel magnetic fields composing the quadrupole magnetic field. The directionality in the upper and lower regions of the quadrupole magnetic field was opposite to that in the inner and outer regions.
\end{abstract}

PACS numbers: 52.20.-j Elementary processes in plasma, 52.25.Fi Transport properties 
Published source: Plasma Sources Science and Technology 20(6) (2011) 065003 (7 pages)

Structure and dynamics of NLD plasma

\section{Introduction}

The requirements for anisotropic fine etching and a high throughput in dry processes in semiconductor fabrication have driven the development of low-pressure plasma sources. The magnetic neutral loop discharge (NLD) plasma proposed by Uchida (1994) is one of the most promising plasma operation modes for satisfying these requirements.

The NLD plasma is a type of inductively coupled magnetized plasma. Uchida and Hamaguchi (2008) presented a substantial overview of the features and applications of NLD plasmas. A typical NLD plasma reactor is surrounded by three coaxial coils (top, middle and bottom coils) to apply a characteristic quadrupole magnetic field to the plasma. The current of the middle coil flows in the opposite direction to those of the top and bottom coils. Using this arrangement a ring-shaped region of zero magnetic field, the so-called neutral loop (NL), is formed in the reactor. The NLD plasma is generated along the NL by an electric field induced by a radio-frequency (rf) antenna. The magnetic field has the dual function of confining electrons around the NL and inducing a partial electron cyclotron resonance. This allows an efficient power deposition to the electrons, which sustains a high plasma density even at low gas pressures; e.g., $10^{10}-10^{11} \mathrm{~cm}^{-3}$ in Ar at $0.067-0.13 \mathrm{~Pa}$ (Tsuboi et al 1995, 1997).

Another remarkable feature of the NLD plasma is that the radius and position of the NL are adjustable by the coil currents; this feature could be used for uniform wide-area processing. Chen et al (1999) obtained uniform etching rate distributions for $200 \mathrm{~mm}$ wafers with $2-3 \%$ deviations of $3 \sigma$ by temporally varying the NL radius at a repetition frequency of $0.1 \mathrm{~Hz}$. Sugawara et al (2010) demonstrated that the transport of energetic electrons and the resulting generation of reactive species occur along the separatrices of the quadrupole magnetic field, and inferred that the etching rate would be high at the foot of the separatrices. On the basis of this result, Sugawara (2010) proposed a strategy to let the foot of the separatrix sweep over the substrate to make the time-averaged etchant flux on the substrate uniform; a uniform etchant flux distribution would be obtained by making an ensemble of those at various NL radii 
Published source: Plasma Sources Science and Technology 20(6) (2011) 065003 (7 pages)

Structure and dynamics of NLD plasma

with appropriately chosen weights of the etchant irradiation time. Sakurai et al (2011) demonstrated a uniform ensemble distribution of ion flux on the substrate obtained with this method.

In this paper, we analyse the structure and rf response of a $\mathrm{CF}_{4}$ NLD plasma using a Monte Carlo method. In addition to the spatial distributions of the electron number density and the mean electron energy, which have often been analysed in conventional investigations, those of the electron energy gain and the azimuthal electron flux are calculated in order to understand the dynamics of the NLD plasma. These quantities will be informative when we interpret results of macroscopic observations and/or analyses of NLD plasma response. For example, Sugawara et al (2011) mentioned that the asymmetry in the phase-resolved optical emission in a Ne NLD plasma reported by O'Connell et al (2008) might be an appearance of the rectification effect of the antiparallel magnetic field on the rf response of the electron energy gain. Also, the profile of the loop current of NLD plasmas would give an orientation of the extension to the equivalent circuit model proposed by Tsuboi and Ogata (2007) to evaluate the plasma power. The dynamics of the NLD plasma is investigated from the viewpoint of the electron motion in a quadrupole magnetic field.

\section{Simulation model}

\subsection{Reactor geometry and electric and magnetic fields}

We assume the geometry shown in figure 1, i.e., that of a model NLD plasma reactor. The chamber is a cylinder with a $400 \mathrm{~mm}$ inner diameter and a $400 \mathrm{~mm}$ height. We take the $r$ and $z$ coordinates, respectively, as the radial and axial positions relative to the origin at the centre of the cylinder. Three magnetic coils to form the static magnetic field $\boldsymbol{B}$ and a one-turn rf antenna to induce the rf electric field $\boldsymbol{E}$ having a sinusoidal waveform with a frequency $f=13.56 \mathrm{MHz}$ are placed coaxially around the cylinder.

Each coil has 60 turns and the separation between the coils is $120 \mathrm{~mm}$. The dc 


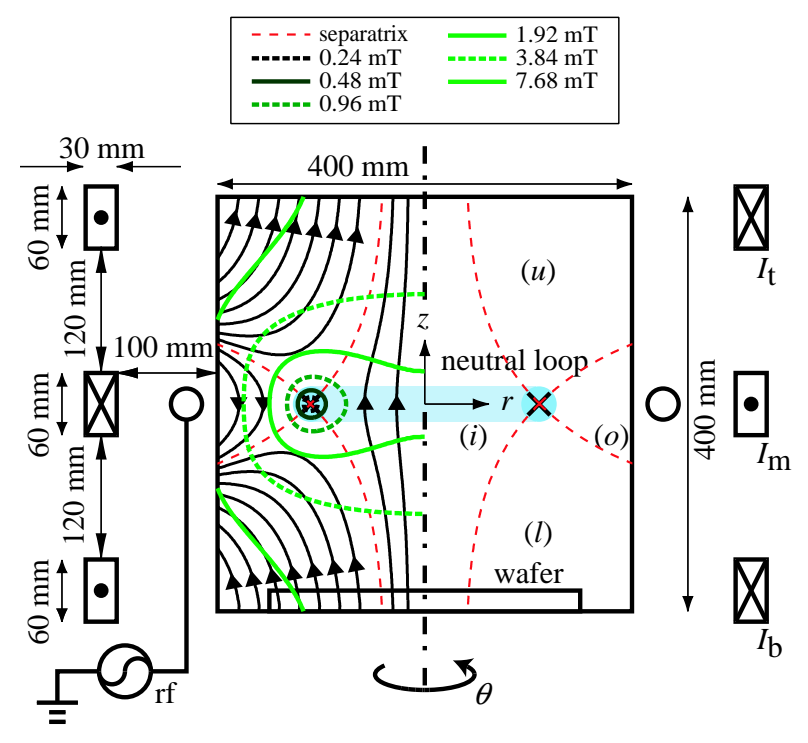

Figure 1. The NLD reactor assumed in this analysis and the quadrupole magnetic field induced by the coil currents $I_{\mathrm{t}}, I_{\mathrm{m}}$ and $I_{\mathrm{b}}$. The X points in the chamber represent the region of null magnetic field (NL) formed at $r=110 \mathrm{~mm}$ and $z=0$. The broken curves crossing at the NL are the separatrices. The four regions labelled $(i),(o),(u)$ and $(l)$ are named the inner, outer, upper and lower regions, respectively.

coil currents are set as $I_{\mathrm{t}}=+95.6 \mathrm{~A}, I_{\mathrm{m}}=-111.5 \mathrm{~A}$ and $I_{\mathrm{b}}=+95.6$ A. The $\boldsymbol{B}$ field in the reactor calculated on the basis of Biot-Savart's law in this condition is shown also in figure 1. The NL is formed at $r=110 \mathrm{~mm}$ and $z=0$. The $\boldsymbol{B}$ field around the NL is quadrupole, and its gradient is $0.36 \mathrm{mT} \mathrm{cm}^{-1}$. The broken curves crossing at the NL are the separatrices, which represent the boundary between the four regions of the quadrupole $\boldsymbol{B}$ field. Hereafter, we call the four regions the inner, outer, upper and lower regions. In addition, we define the NL region as the torus-like region around the NL, in which $|\boldsymbol{B}| \leq 2 B_{\mathrm{ECR}}$. Here, $B_{\mathrm{ECR}}=0.48 \mathrm{mT}$ is the strength of the rf-resonant $\boldsymbol{B}$ field at $f=13.56 \mathrm{MHz}$. Its value is derived from the condition of the electron cyclotron resonance as $B_{\mathrm{ECR}}=2 \pi f m / e$, where $e$ and $m$ are the electronic charge and mass.

The ratio between the coil currents determines the NL position, and their absolute values determine the gradient. While the NL is often formed near the side wall, in practical operations for a tight electro-magnetic coupling with the rf antenna, the NL 
Published source: Plasma Sources Science and Technology 20(6) (2011) 065003 (7 pages)

Structure and dynamics of NLD plasma

may be moved inward for uniform processing. In the present simulation, the NL is formed apart from the side wall so that we can observe the electron motion in both of the inner and outer regions. In addition, the gradient of the $\boldsymbol{B}$ field, on which the thickness of the NL region is dependent, is chosen to observe the directionality in the azimuthal electron conduction around the NL region as shown in section 3.4.

The amplitude of the rf antenna current is set at $30 \mathrm{~A}$. The $\boldsymbol{E}$ field induced in the chamber is calculated on the basis of Faradey's law of induction assuming charge-free space. At this time, the amplitude of the azimuthal component $E_{\theta}$ of the $\boldsymbol{E}$ field is $4.0 \mathrm{~V} \mathrm{~cm}^{-1}$ at the NL. We neglect the skin effect of the plasma, the space charge field with radial and axial components $E_{r}$ and $E_{z}$, and the magnetic fields induced by the rf antenna and the loop plasma current itself. We focus here on the essential action of the quadrupole $\boldsymbol{B}$ field to govern the electron motion.

\subsection{Monte Carlo method}

The electron motion in the NLD plasma is simulated by a Monte Carlo method. The Runge-Kutta fourth-order method is applied to the equations of motion, $\mathrm{d} \boldsymbol{x} / \mathrm{d} t=\boldsymbol{v}$ and $\mathrm{d} \boldsymbol{v} / \mathrm{d} t=-(e / m)(\boldsymbol{E}+\boldsymbol{v} \times \boldsymbol{B})$. Here, $\boldsymbol{x}=(x, y, z)\left(r=\sqrt{x^{2}+y^{2}}\right)$ and $\boldsymbol{v}=\left(v_{x}, v_{y}, v_{z}\right)$ represent the position and velocity of an electron, respectively.

$\mathrm{CF}_{4}$, which is an etching gas, is chosen as the medium. The gas molecule number density is set at $1.77 \times 10^{14} \mathrm{~cm}^{-3}$, which corresponds to a gas pressure $0.67 \mathrm{~Pa}(=$ 5.0 mTorr) at $0^{\circ} \mathrm{C}$. The electron collision cross sections of $\mathrm{CF}_{4}$ are taken from Kurihara et al (2000). They include the processes of momentum transfer, excitation, neutral dissociation, and dissociative ionization and attachment. Only two-body interactions between electrons and $\mathrm{CF}_{4}$ molecules are considered in the simulation. The timesaving scheme for judgement of electron-molecule collisions (Sugawara et al 2007) is adopted.

The initial electrons are supplied from the NL region, with velocities chosen at random from a Maxwellian distribution with a mean energy of $1 \mathrm{eV}$, and their motion under the $\boldsymbol{E}$ and $\boldsymbol{B}$ fields are traced for $300 \mathrm{rf}$ periods. Since the electron reflectivity at 
Published source: Plasma Sources Science and Technology 20(6) (2011) 065003 (7 pages)

Structure and dynamics of NLD plasma

the chamber wall is unknown, we assume a high value of $99 \%$ for it, representing the total electron-surface interactions at the boundary following the discussion by Sugawara et al (2010). The electron reflection can act as an alternative function to the space charge field to retain the electron population in the chamber.

We calculate the distributions of the mean electron energy $\bar{\varepsilon}(r, z)$, electron number density $N(r, z)$, the electron energy gain $G(r, z)$ and the azimuthal electron flux $\Phi(r, z)$ by sampling electrons with spatial resolutions of $\Delta r=5 \mathrm{~mm}$ and $\Delta z=10 \mathrm{~mm}$. In order to reduce the statistical fluctuation, we sample the electrons after the initial relaxation process of $200 \mathrm{rf}$ periods and superpose the distributions over the last $100 \mathrm{rf}$ periods of the simulation time.

\section{Results and discussion}

\subsection{Electron trajectory}

Figure 2 shows a typical electron locus in the quadrupole $\boldsymbol{B}$ field. Three elementary motions are observed. The first is a meandering along the NL, by which electrons gain energy from the $\boldsymbol{E}$ field. The second is gyration around a magnetic field line induced by the Lorentz force. The third is a reciprocating motion along the magnetic field line. Electrons move away from the NL and turn back by the magnetic mirror effect or electron reflection at the chamber wall.

Because the electron diffusion perpendicular to the magnetic field lines is limited at the present gas pressure and in the present $\boldsymbol{B}$ field, the gyration and the reciprocating motion are the dominant processes of electron transport to form the structure of the NLD plasma.

\subsection{Electron distribution}

Figure 3 shows the electron number density $N(r, z)$ normalized by its peak value. More than $10^{7}$ electrons were sampled effectively. 

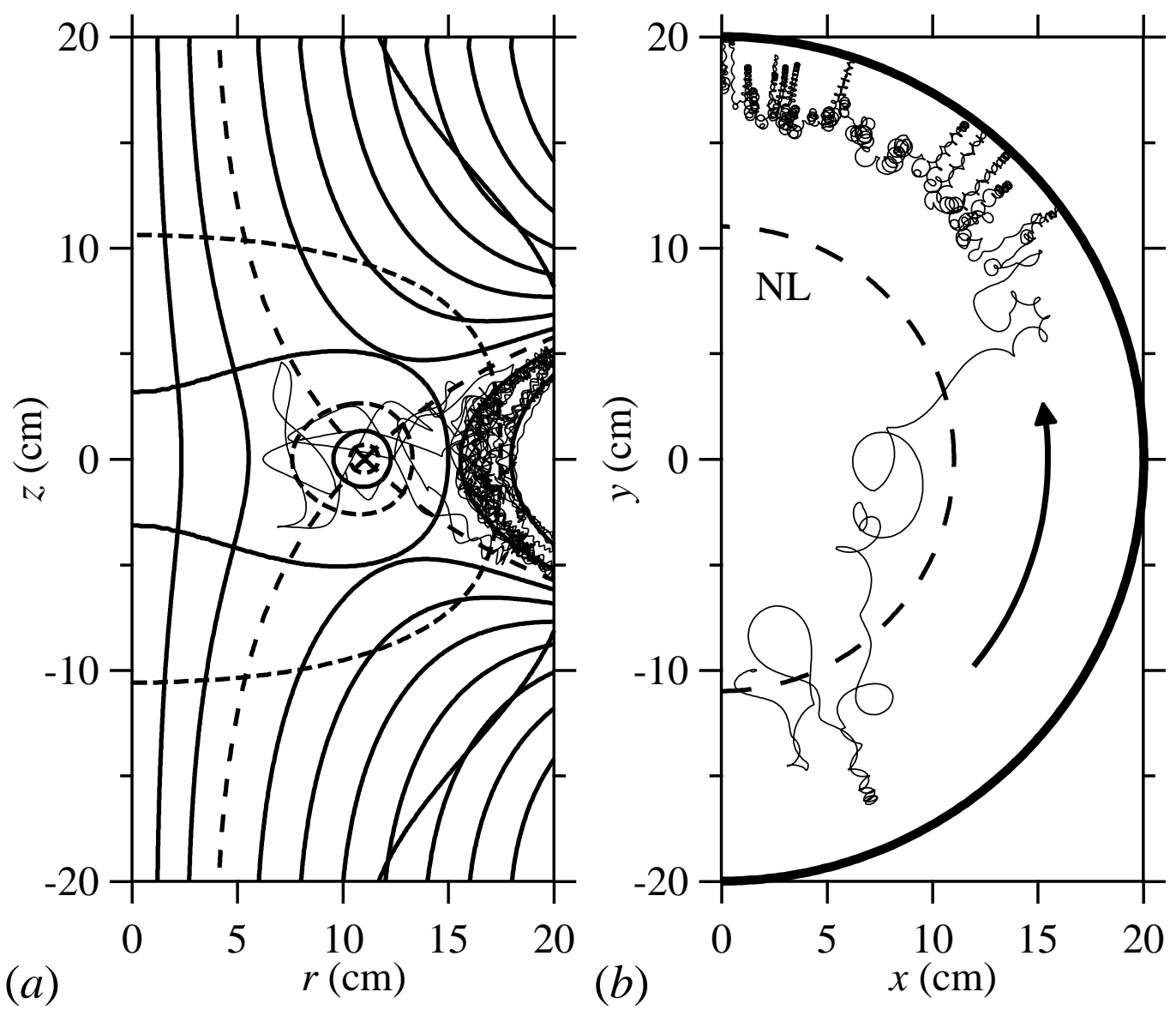

Figure 2. Typical electron motion under the $\operatorname{rf} \boldsymbol{E}$ field and the quadrupole $\boldsymbol{B}$ field assumed in the simulation: $(a) r-z$ plot (side view) and (b) $x-y$ plot (top view). The separatrices and the NL are drawn together.

$N(r, z)$ has valleys along the separatrices and ridges beside them. Vural and Brinkmann (2007) reported a similar structure in a capacitively coupled NLD plasma and elaborated on the mechanism to form it, referring to the trapping effect of the magnetic mirror discussed by Okraku-Yirenkyi et al (2001). Here, we explain this structure from the viewpoint of single-electron motion with a concept of 'guiding magnetic field lines' around which electrons gyrate and along which they undergo the reciprocating motion. Those electrons for which the guiding magnetic field line passes through the NL region have the opportunity of acceleration by the $\boldsymbol{E}$ field when they enter the NL region during their reciprocating motion. In the weak $\boldsymbol{B}$ field near the NL, gyration does not occur. The electrons meander and, owing to the energy gained from 


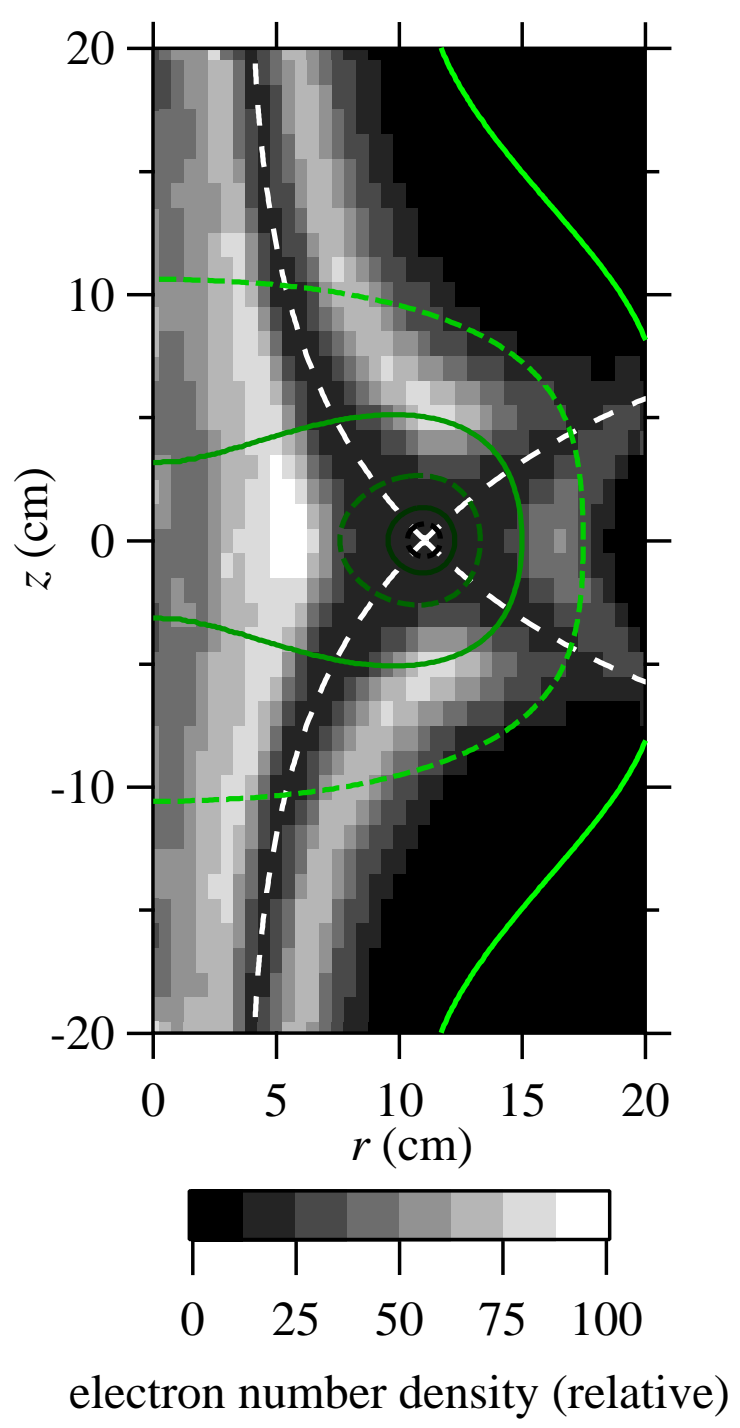

Figure 3. The electron distribution $N(r, z) . N(r, z)$ has valleys along the separatrices, and ridges are formed beside them.

the acceleration, they eventually transfer their guiding magnetic field lines to others which do not pass through the NL region. This transfer moves the electrons away from the separatrices (Osaga et al 2011).

Electrons away from the separatrices have little opportunity for acceleration under the strong $\boldsymbol{B}$ field. After an electron loses its energy by inelastic collisions, which occurs eventually, it keeps the reciprocating motion along a guiding magnetic field line with a low electron energy. The transfer of guiding magnetic field line may occur also by an elastic collision, which appears as the electron diffusion. However, the collision frequency 
Published source: Plasma Sources Science and Technology 20(6) (2011) 065003 (7 pages)

Structure and dynamics of NLD plasma

is low in the present condition, and the displacement of the guiding magnetic field line is small when the electron energy is low, so further electron diffusion hardly occurs. The electron accumulation by this process formed the ridge of $N(r, z)$ beside the separatrices. This profile seems to be a characteristics of a $\boldsymbol{B}$ field-dominated case under a relatively strong $\boldsymbol{B}$ field. A similar off-separatrix peak of the electron distribution is seen in an experimental result of Sugawara et al (2010) as well.

\subsection{Electron energy gain and consumption}

Figure 4 shows the distribution of the mean electron energy $\bar{\varepsilon}(r, z)$. High values of $\bar{\varepsilon}(r, z)$ are distributed not only in the NL region, where the energy gain of electrons occurs, but also around the separatrices. These regions correspond to the valleys of $N(r, z)$. This is because the low-energy electrons mentioned in the preceding subsection accumulate besides the separatrices and reduce the mean electron energy.

Details of the energy transport can be observed in the distribution of the electron energy gain and consumption. Figure 5 shows the distribution of the time-averaged net electron energy gain per unit time, $G(r, z)$, in each sampling section $\Delta r \Delta z$. G(r,z) includes the energy gain and loss of electrons due to the acceleration and deceleration by the $\boldsymbol{E}$ field (positive and negative values, respectively) and the energy consumption by inelastic collisions (negative value). The energy transport accompanying the movement of electrons from one sampling section to another is not included in $G(r, z)$. That is, $G(r, z)$ represents the energy inlet and outlet of the NLD plasma.

In the NL region, the energy gain exceeds the loss. This agrees with the conventional understanding that the electron energy gain occurs in the NL region. In contrast, the region in which the energy consumption occurs is distributed along the separatrices. This represents the energy flow from the NL region along the separatrices. The distribution of the ionization rate $R_{\mathrm{i}}(r, z)$ in figure 6 , most of which is of dissociative ionization $\mathrm{CF}_{4}+\mathrm{e} \rightarrow \mathrm{CF}_{3}^{+}+\mathrm{F}+2 \mathrm{e}$ (threshold: $16.0 \mathrm{eV}$ ), shows that the reactions to produce etchant species occur not only in the NL region but also along the separatrices. The 


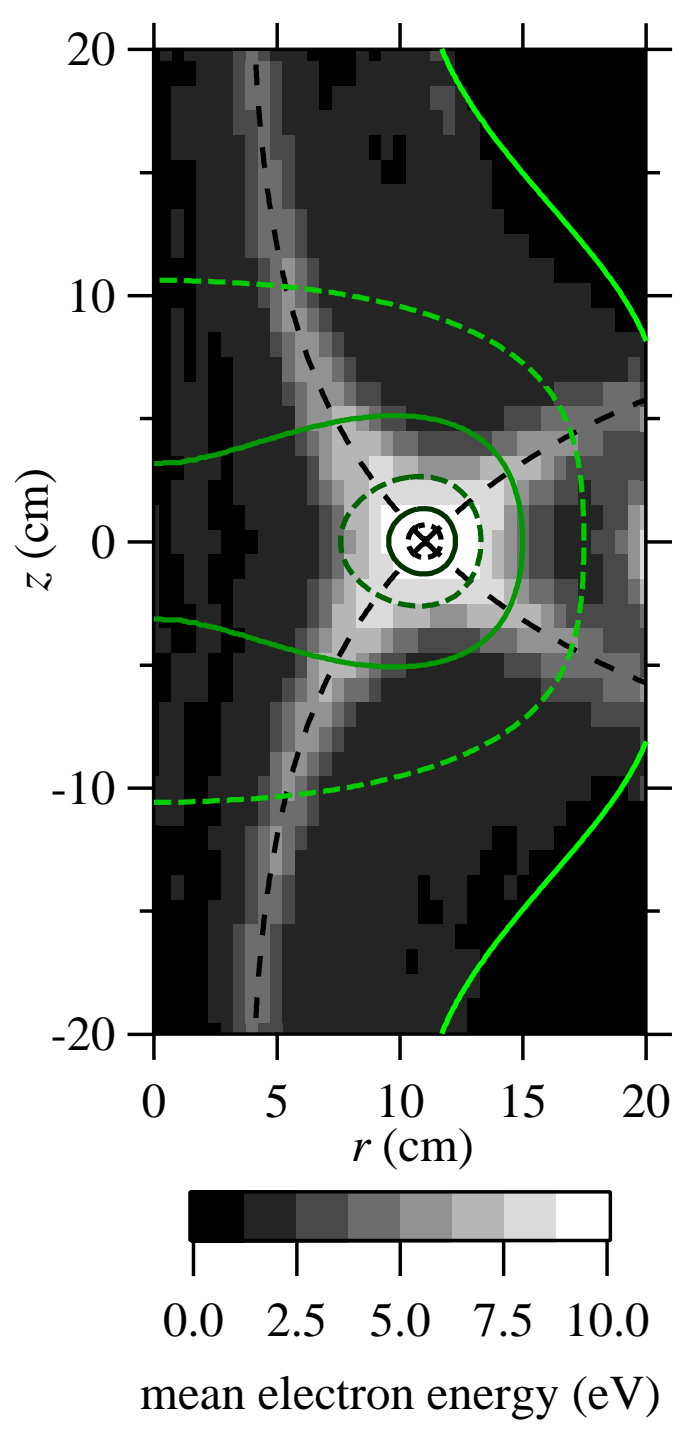

Figure 4. Distribution of the mean electron energy $\bar{\varepsilon}(r, z)$. The value of $\bar{\varepsilon}(r, z)$ is high at the NL and on the separatrices.

distribution of $R_{\mathrm{i}}(r, z)$ resembles $\bar{\varepsilon}(r, z)$ rather than $N(r, z)$. The ionization is more dependent on the electron energy than on the electron population.

\subsection{Azimuthal electron flux}

Figure 7 shows the time-averaged azimuthal electron flux $\Phi(r, z)$, which represents the $\theta$ component of the electron flow to form the loop plasma current. Here, $\theta$ satisfies $x=r \cos \theta$ and $y=r \sin \theta$. Positive and negative values of $\Phi(r, z)$, respectively, represent anticlockwise and clockwise electron motions relative to the central axis of the chamber 


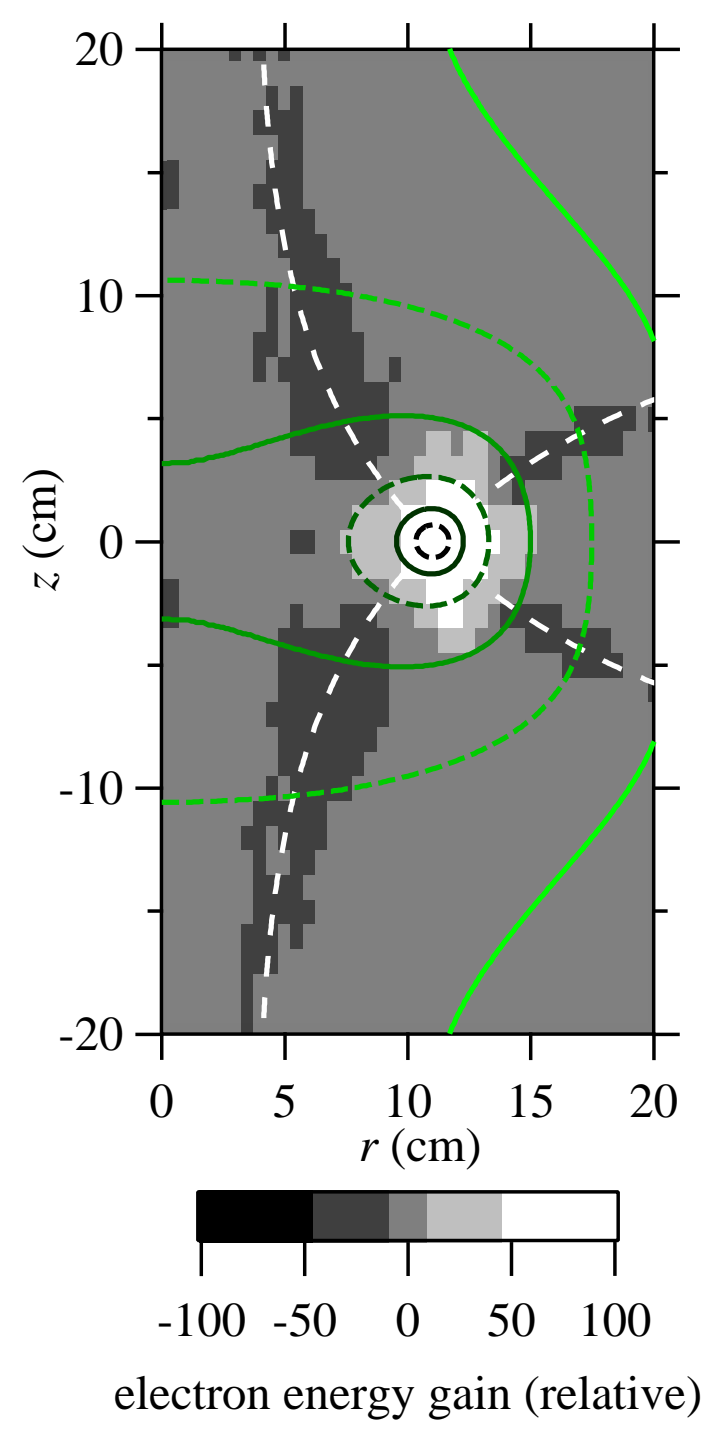

Figure 5. Distribution of the time-averaged electron energy gain $G(r, z)$. Positive and negative values represent the power deposition from the rf antenna and the electron energy loss by inelastic collisions, respectively.

in the top view.

$\Phi(r, z)$ indicates that the clockwise and anticlockwise electron motions are temporally asymmetric and its distribution has a structure. In the inner and outer regions, the electron flux is anticlockwise near the NL and tends to be clockwise at farther positions. In the upper and lower regions, the direction is inverse.

This directionality arises from the rectification effect of antiparallel $\boldsymbol{B}$ fields discussed by Sugawara and Sakai (2008) and Sugawara et al (2011). The quadrupole 


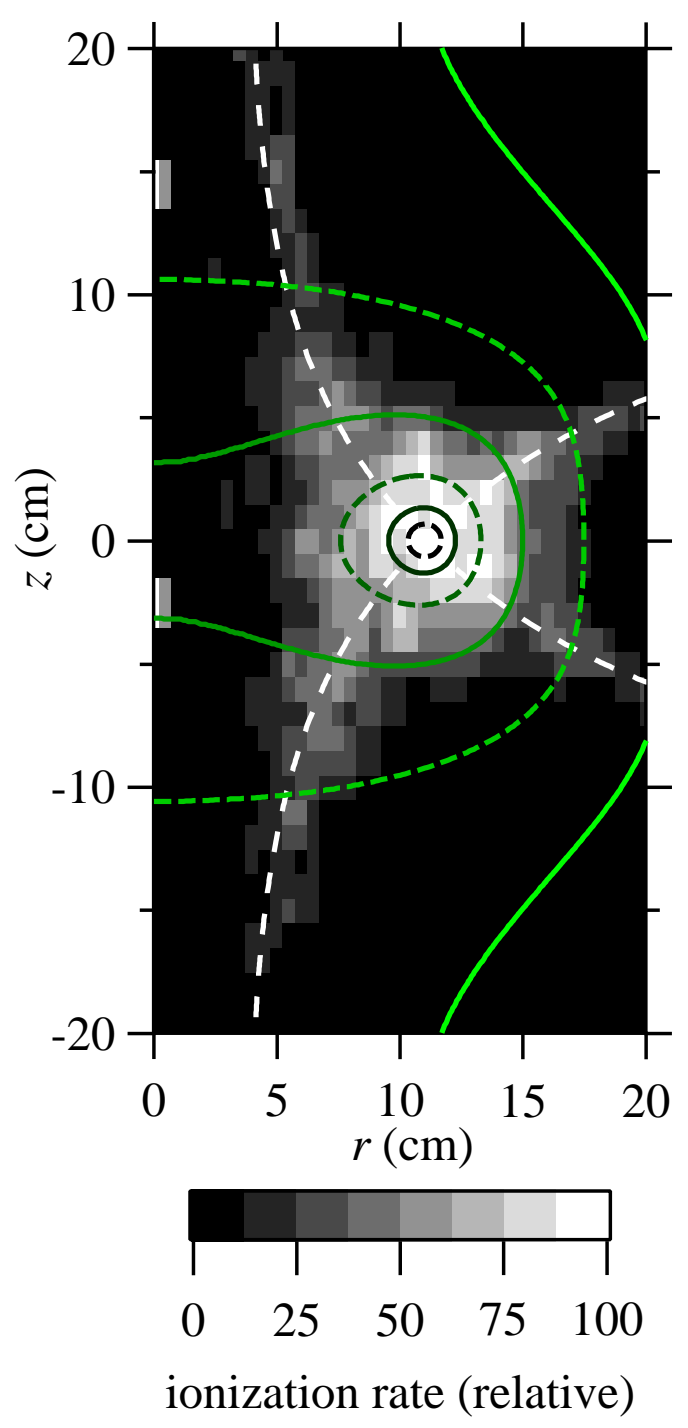

Figure 6. Distribution of the time-averaged ionization rate $R_{\mathrm{i}}(r, z)$.

$\boldsymbol{B}$ field also has the rectification effect because it consists of two pairs of antiparallel $\boldsymbol{B}$ fields. Here, we see that a gyrating electron in the outer region drifted anticlockwise (towards the $+\theta$ direction) in figure $2(b)$, but $\Phi(r, z)<0$ on the far side from the NL in the outer region (figure 7). This seemingly paradoxical result has been convincingly explained from the gradient of $N(r, z)$ in Sugawara et al (submitted); gyration involves both clockwise and anticlockwise motions when observed from the central axis of the chamber, which correspond, respectively, to the negative and positive fluxes, and their local sum can be negative when $N(r, z)$ decreases outwards. This interpretation is 


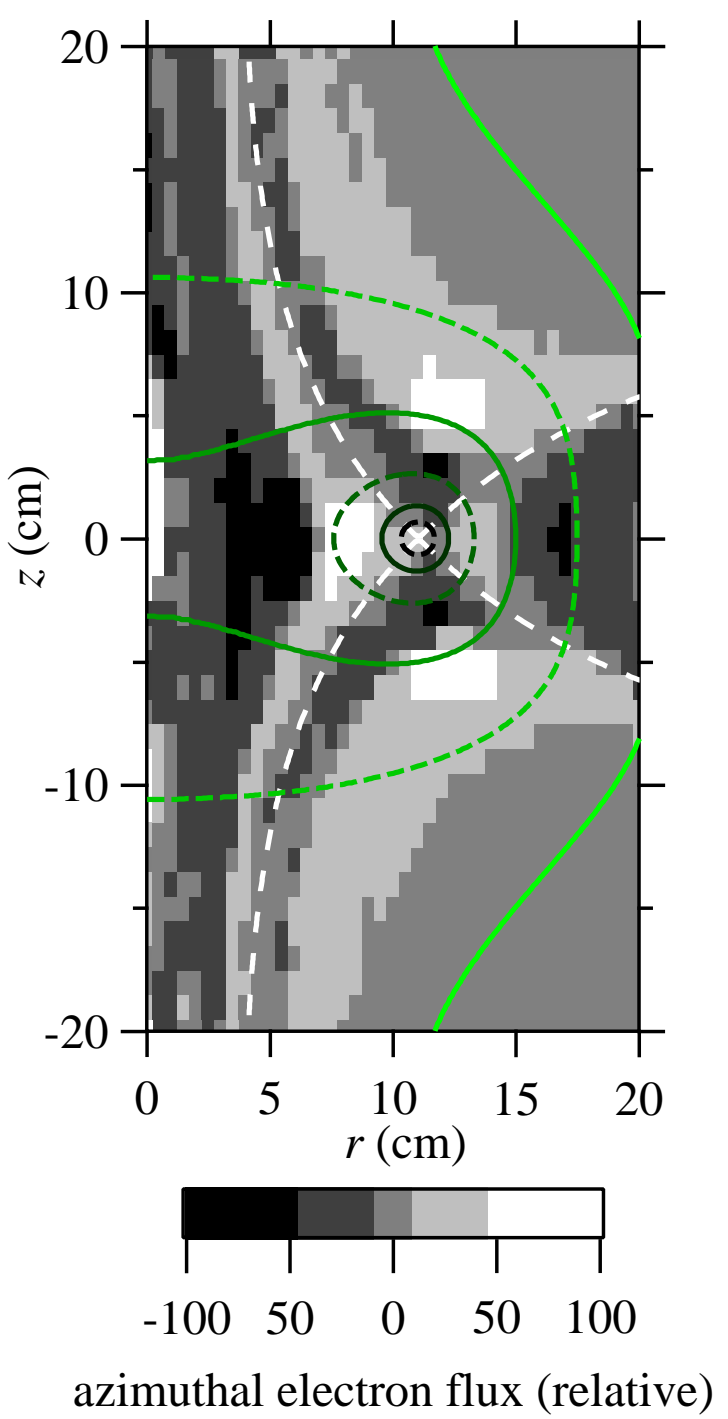

Figure 7. Time-averaged azimuthal electron flux $\Phi(r, z)$.

supported by the result that the border between $\Phi(r, z)>0$ and $\Phi(r, z)<0$ lies along the ridge of $N(r, z)$ in each of the four regions.

Figure 8 shows the rf phase-resolved azimuthal electron flux $\phi(r, z, t)$ on two cross sections $z=0$ and $r=11 \mathrm{~cm}$ crossing at the NL. Here, $\Phi(r, z)=\int_{0}^{T} \phi(r, z, t) \mathrm{d} t / T$ and $T=1 / f$. At the NL, the alternation of $\phi(r, z, t)$ follows that of $E_{\theta}(r, z, t)=$ $E_{\theta}(r, z, 0) \sin 2 \pi f t$ with a slight delay, where $E_{\theta}(r, z, 0)>0$. Its waveform is symmetric between one half of the rf period and the other. As the distance from the NL increases, the phase of $\phi(r, z, t)$ increasingly leads. This tendency is common between 


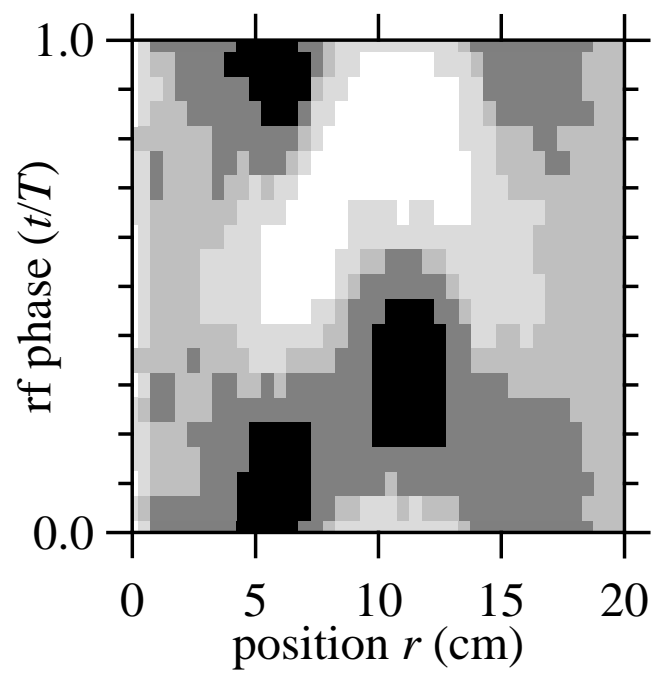

(a)

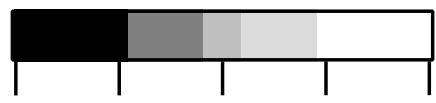
$\begin{array}{lllll}-100 & -50 & 0 & 50 & 100\end{array}$ azimuthal electron flux (relative)

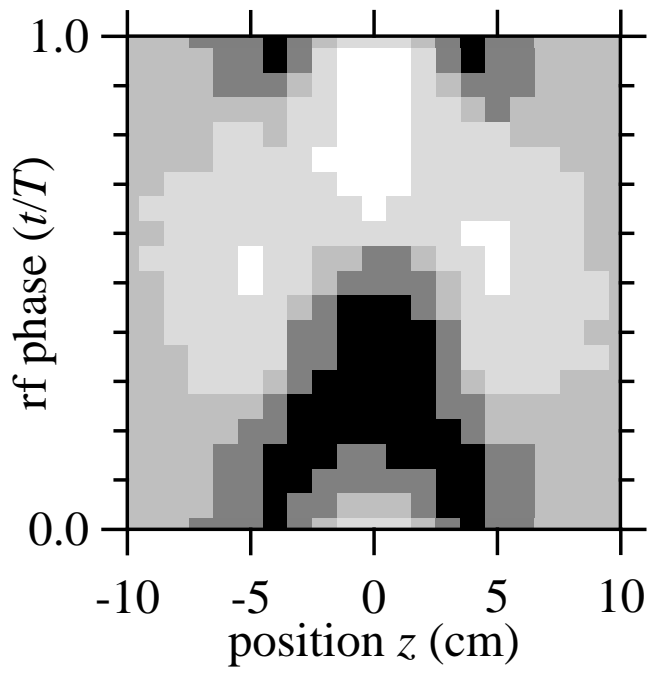

(b)
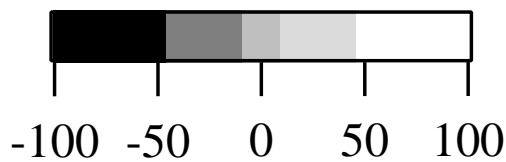
azimuthal electron flux (relative)

Figure 8. Streak images of the rf phase-resolved azimuthal electron flux $\phi(r, z, t)$ at $(a) z=0$ (the inner and outer regions) and $(b) r=11 \mathrm{~cm}$ (the upper and lower regions). The NL is at $r=11 \mathrm{~cm}$ and $z=0$.

the inner/outer regions (figure 8(a)) and the upper/lower regions (figure 8(b)). However, because of the rectification effect, slight biases with opposite signs are observed: positive in the former and negative in the latter.

\subsection{Appearance of the rectification effect}

Figure 9 shows the rf phase-resolved response of $\bar{\epsilon}(r, z, t) n(r, z, t)$ on the same cross sections as figure 8. Here, $\bar{\epsilon}(r, z, t)$ and $n(r, z, t)$ are instantaneous values of the mean electron energy and the electron number density, respectively, where $\bar{\varepsilon}(r, z)=$ $\int_{0}^{T} \bar{\epsilon}(r, z, t) \mathrm{d} t / T$ and $N(r, z)=\int_{0}^{T} n(r, z, t) \mathrm{d} t / T$. The peaks of $\bar{\epsilon}(r, z, t) n(r, z, t)$ in the inner and outer regions in figure $9(a)$ appear around $r=7$ and $15 \mathrm{~cm}$ at $t / T=0.0$, while those in the upper and lower regions in figure $9(b)$ appear around $z= \pm 4 \mathrm{~cm}$ at $t / T=0.5$. Note here that $\bar{\epsilon}(r, z, t) n(r, z, t)$ has its peaks only once per rf period although $\left|E_{\theta}\right|$ peaks twice. 


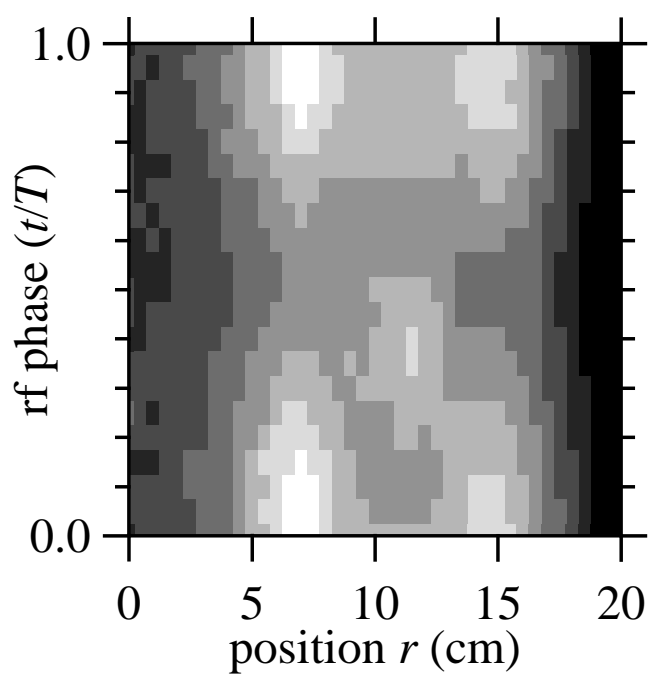

(a)

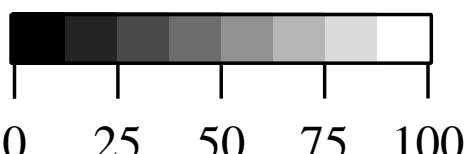
electron energy density (relative)

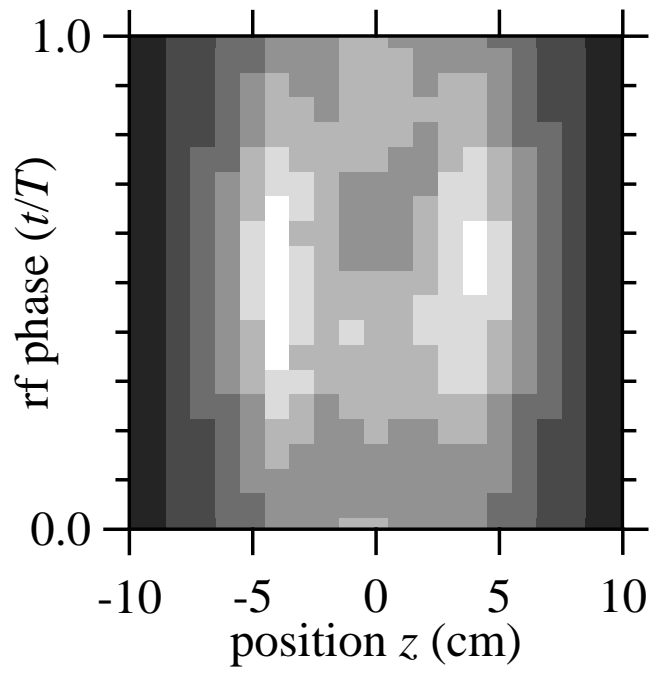

(b)

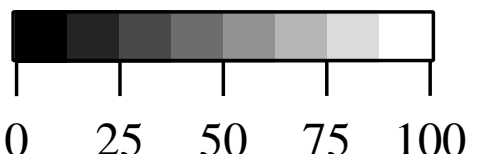
electron energy density (relative)

Figure 9. Streak images of the rf phase-resolved energy density $\bar{\epsilon}(r, z, t) n(r, z, t)$ at $(a)$ $z=0$ (the inner and outer regions) and $(b) r=11 \mathrm{~cm}$ (the upper and lower regions). The NL is at $r=11 \mathrm{~cm}$ and $z=0$.

A similar asymmetric profile is seen in an experimental result by O'Connell et al (2008). They reported a phase-resolved waveform of optical emission from a Ne NLD plasma measured at the centre of the NL. The waveform was asymmetric between one half of the rf period and the other, while it was symmetric when no $\boldsymbol{B}$ field was applied. Since the intensity of the optical emission is dependent on both the electron energy and population, it would be reasonable to relate the profiles of $\bar{\epsilon}(r, z, t) n(r, z, t)$ and the emission.

In order to clarify the overall mechanism by which the asymmetry appeared in the experiment, further development of the simulation model would be required. For example, we have to consider the space charge field, the distribution of the excitation collisions with optical emission, and the interaction between the plasma current and the external circuit. A currently available technique to examine the asymmetry due to the rectification effect would be the equivalent circuit analysis, which was performed by 
Published source: Plasma Sources Science and Technology 20(6) (2011) 065003 (7 pages)

Structure and dynamics of NLD plasma

Tsuboi and Ogata (2007) for an Ar NLD plasma. The NLD plasma was modelled as a ring conductor in the analysis. A possible customization would be to treat the NLD plasma as parallel ring conductors. The rectification effect would be taken into account by inserting diodes into the ring conductors in series, and other factors characterizing the four regions of the quadrupole $\boldsymbol{B}$ field, such as the volume, the electron population and the distance from the rf antenna, would be involved in the resistance and mutual inductance of the ring conductor. These efforts would be implemented in future.

\section{Conclusion}

In order to analyse the structure and dynamics of an NLD plasma, electron motion in the quadrupole $\boldsymbol{B}$ field was simulated by a Monte Carlo method.

The spatial distributions of the electron number density $N(r, z)$ had valleys along the separatrices of the quadrupole $\boldsymbol{B}$ field and ridges beside them. The mechanism of forming the structure of $N(r, z)$ was explained from the transfer of electrons between guiding magnetic field lines due to the electron acceleration in the NL region. The mean electron energy $\bar{\varepsilon}(r, z)$ was high, not only in the NL region, but also along the separatrices. Analysis on the net electron energy gain $G(r, z)$ confirmed that the electrons gained energy from the $\boldsymbol{E}$ field in the NL region. In addition, the electric power given to the electrons was transported along and consumed around the separatrices.

The azimuthal electron flux $\Phi(r, z)$ showed a directionality of the electron flow in the $\theta$ direction. It was demonstrated that the rectification effect of the antiparallel $\boldsymbol{B}$ field discussed in Sugawara et al (2011) also appears in a practical quadrupole $\boldsymbol{B}$ field in the NLD plasma.

The present simulation model took account of only essential factors governing the electron motion in the NLD plasma. In subsequent work to establish an entire simulation model which can reproduce or predict responses of NLD plasmas in detail, we need to consider many practical factors such as the gaseous chemistry (including positive and negative ions and radicals), the gas flow dynamics at low pressures, and the plasma- 
Published source: Plasma Sources Science and Technology 20(6) (2011) 065003 (7 pages)

Structure and dynamics of NLD plasma

surface interactions, as well as the influence of the space charge field and the feedback to the external circuit. Nonetheless, the present attempt has illustrated one of the characteristic features of the NLD plasma. The finding and understanding obtained here provide clues to interpreting experimentally observed phenomena in NLD plasmas resulting from complicated processes.

\section{Acknowledgments}

The authors wish to thank Prof A Murayama of Hokkaido University for valuable comment and kind encouragement. This work was in part supported by ULVAC Inc. and by Grant-in-Aid No. 22540500 from the Japan Society for the Promotion of Science.

\section{References}

Chen W, Morikawa Y, Ito M, Hayashi T, Sugita K, Shindo H and Uchida T 1999 J. Vac. Sci. Technol. A $172546-50$

Kurihara M, Petrović Z Lj and Makabe T 2000 J. Phys. D: Appl. Phys. 33 2146-53

O'Connell D, Gans T, Crintea D L, Czarnetzki U and Sadeghi N 2008 Plasma Sources Sci. Technol. 17024022

Okraku-Yirenkyi Y, Sung Y-M, Otsubo M, Honda C and Sakoda T 2001 J. Vac. Sci. Technol. A 19 $2590-5$

Osaga T, Sakurai Y and Sugawara H 2011 IEEE Trans. Plasma Sci. 39 in press (DOI: 10.1109/TPS.2011.2151882)

Sakurai Y, Osaga $\mathrm{T}$ and Sugawara H 2011 IEEE Trans. Plasma Sci. 39 in press (DOI: 10.1109/TPS.2011.2128895)

Sugawara H 2010 Proc. 63rd Ann. Gaseous Electronics Conf. and 7th Int. Conf. Reactive Plasmas (Paris, France, 4-8 October) DTP.27

Sugawara H, Mori N, Sakai Y and Suda Y 2007 J. Comp. Phys. 223 298-304

Sugawara H, Osaga T, Tsuboi H, Kuwahara K and Ogata S 2010 Japan. J. Appl. Phys. 49086001

Sugawara H, Osaga T and Yamamoto H 2011 Plasma Sources Sci. Technol. 20055002

Sugawara H and Sakai Y 2008 J. Phys. D: Appl. Phys. 41135208

Tsuboi H, Hayashi T and Uchida T 1997 Japan. J. Appl. Phys. 36 6540-4

Tsuboi H, Itoh M, Tanabe M, Hayashi T and Uchida T 1995 Japan. J. Appl. Phys. 34 2476-81 
Published source: Plasma Sources Science and Technology 20(6) (2011) 065003 (7 pages)

Structure and dynamics of NLD plasma

Tsuboi H and Ogata S 2007 Japan. J. Appl. Phys. 46 7475-7

Uchida T 1994 Japan. J. Appl. Phys. 33 L43-4

Uchida T and Hamaguchi S 2008 J. Phys. D: Appl. Phys. 41083001

Vural M and Brinkmann R P 2007 J. Phys. D: Appl. Phys. 40 510-9 